\title{
Um estudo de caso sobre um Teste Adaptativo baseado no conhecimento tácito do professor sob o ponto de vista da motivação do estudante
}

\author{
Sérgio R. I. Yoshioka ${ }^{1}$, Alexandre Teixeira ${ }^{1}$, Lucila Ishitani ${ }^{1}$ \\ ${ }^{1}$ Instituto de Ciências Exatas e Informática \\ Pontifícia Universidade Católica de Minas Gerais (PUC Minas) \\ Rua Walter Ianni, 255 - São Gabriel - Belo Horizonte/MG - CEP: 31980110 \\ sergio.yoshioka@sga.pucminas.br, \{teixeira, lucila\}@pucminas.br
}

\begin{abstract}
This paper describes a case study about a Stratified Adaptive Test, based on the tacit knowledge of the professor and applied in two classes of Computer Network. Data were collected about student's motivation after answering five exercises. In data analysis, we sought to identify correlations between motivation factors, and also some behavior that stood out. Our results present correlation between Interest and three another factors: Probability of Success, Challenge and Dedication Time. Besides that, although the results didn't achieve the expected for a Computer Adaptive Test (CAT), we found some benefits from the point of view of student's motivation, which indicates this method is recommended for classroom, considering the ease of implementation.
\end{abstract}

Resumo. Este artigo descreve um estudo de caso sobre um Teste Adaptativo Estratificado, baseado no conhecimento tácito do professor e aplicado em duas turmas de Redes de Computadores. Foram coletados dados relacionados à motivação dos alunos, após a execução de cinco exercícios. Na análise de dados, buscou-se identificar correlações entre os fatores de motivação e, também, algum comportamento que se destacasse. Os resultados apresentaram correlação entre o fator Interesse e outros três fatores: Probabilidade de Sucesso, Desafio e Tempo de Dedicação. Além disso, apesar de não atingirem o esperado para um Teste Adaptativo Informatizado (TAI), apresentaram benefícios do ponto de vista da motivação do estudante, o que indica ser recomendável a utilização da metodologia em sala de aula, considerando-se sua facilidade de implementação.

\section{Introdução}

Os avanços das tecnologias da informação têm transformado significativamente diversas áreas do conhecimento, incluindo a educação. No contexto atual, os tradicionais modelos de ensino estão dando lugar a novas metodologias em que o aluno passa a ser peça ativa no seu próprio aprendizado, o que cria uma grande diversidade de estudantes, seja no perfil, no nível de conhecimento, nos interesses e nas curiosidades. Para melhor aproveitar este potencial, cada vez mais se exige do professor o papel de orientador, que usa da sua experiência para indicar aos seus alunos bons caminhos, deixando-os livres 
V Congresso Brasileiro de Informática na Educação (CBIE 2016)

Anais do XXVII Simpósio Brasileiro de Informática na Educação (SBIE 2016)

para explorar o espaço do conhecimento; ao contrário da figura centralizadora que padronizava, restringia e selecionava o conteúdo que seria exposto. Entretanto, este é um papel muito difícil e trabalhoso de se exercer. Assim, para apoiar o professor, é interessante desenvolver ferramentas inteligentes, que se adaptem individualmente a cada aluno, identifiquem seus pontos fracos e fortes e os reportem.

Dentre essas ferramentas, o Teste Adaptativo Informatizado (TAI) vem ganhando notoriedade nos últimos anos. Ele consiste de um teste computadorizado que se adapta ao conhecimento do usuário, buscando reduzir o tédio ou a frustração do estudante e avaliando-o de forma mais rápida e precisa do que os testes tradicionais (que oferecem o mesmo conjunto de questões para todos os avaliados) [Meijer and Nering 1999]. Uma maneira comum de se implementar um TAI é aplicando-se a Teoria de Resposta ao Item (TRI), pois torna possível que os estudantes sejam avaliados e comparados mesmo respondendo a questões distintas. Entretanto, é necessária uma calibração para que se conheçam os parâmetros das questões. Para isso, são utilizados testes pilotos e técnicas estatísticas que devem ser aplicadas em todas as questões que compõem o banco de questões do TAI, muitas vezes requerendo equipes especializadas em estatística e computação, trabalho este que deve ser repetido sempre que questões novas são adicionadas ao banco. Todo esse esforço afasta o uso do TAI do dia a dia da sala de aula. Para minimizar esse esforço, algumas abordagens de calibração dinâmica são apresentadas na literatura, como o algoritmo CBAT-2 [Huang 1996] utilizado no CALEAP-Web [Piton-Gonçalves et al. 2009]. Entretanto, o foco deste trabalho está na metodologia adaptativa estratificada proposta em [González-Sacristán et al. 2013], que, apesar de não ser baseada na TRI, tem por objetivo comum melhorar a qualidade da avaliação, ter maior robustez, segurança e relevância das questões apresentadas, além de ser de fácil implementação e aplicação no cotidiano da sala de aula.

Este artigo apresenta um estudo de caso de uma metodologia de seleção de questões [González-Sacristán et al. 2013], com duas turmas da disciplina da área de Redes de Computadores, analisando-se dados colhidos sobre a motivação do estudante (por meio do Questionário de Motivação Corrente [Vollmeyer and Rheinberg 2006]). A motivação é relevante, visto que uma das principais justificativas da utilização de Testes Adaptativos é que estudantes com baixa habilidade não se sintam ansiosos e frustrados e estudantes de alta habilidade não se sintam entediados. Além disso, há indícios na literatura de que a motivação influencia significativamente no resultado do aluno, ao mesmo tempo que são escassos os trabalhos sobre este tema.

Alguns dos resultados encontrados ratificam aqueles apresentados na literatura [Penk and Schipolowski 2015]. Pode-se notar, mesmo nesta abordagem adaptativa estratificada, as correlações entre o Probabilidade de Sucesso pelo estudante e o Resultado, entre o Interesse e o Resultado, além da ausência de correlação entre Ansiedade e Resultado. Mas também são apresentados resultados novos: nota-se que o grupo de estudantes "medianos" apresentaram, em um dos módulos, os maiores valores de Probabilidade de Sucesso, Interesse, Desafio e Ansiedade, indicando que a abordagem tem efeito, mas ainda não supre integralmente o esperado de um TAI. Adicionalmente, foi identificado evidências de correlação entre Interesse e outros três fatores: Probabilidade de Sucesso, Interesse e Tempo.

Este artigo está organizado da seguinte forma: na Seção 2 são apresentados os 
V Congresso Brasileiro de Informática na Educação (CBIE 2016)

Anais do XXVII Simpósio Brasileiro de Informática na Educação (SBIE 2016)

principais trabalhos relacionados; na Seção 3, a metodologia do trabalho; na Seção 4, os resultados obtidos; e, por fim, na Seção 5, as conclusões e as sugestões para continuidade deste trabalho.

\section{Trabalhos Relacionados}

Nesta seção serão apresentados estudos relacionados a motivação do estudante ao responder um teste computadorizado e a proposta de um teste adaptativo estratificado, que melhora a qualidade das avaliações, mantendo a simplicidade de implementação.

\subsection{O Teste e a Motivação do Usuário}

Penk e Schipolowski investigaram expectativa, valor e esforço, suas inter-relações e sua relação com o desempenho em uma avaliação em larga escala [Penk and Schipolowski 2015]. A expectativa se refere a como o estudante nota seu próprio desempenho; o valor, em como ele nota o benefício provindo do teste, como a importância, o prazer (ou autorrealização) e a utilidade; e o esforço é o componente que se refere ao custo, ou quanto esforço é necessário para ser bem sucedido no teste. Para tal, foram utilizados 42.298 estudantes como amostra, com uma média de 15,6 anos ( $\mathrm{SD}=0.61)$.

O teste consistia de 374 itens de matemática e 386 itens de ciências distribuídos em 31 livretos. As medidas de motivação eram coletadas antes e depois dos testes pelo Questionário de Motivação Corrente (QMC) [Freund and Holling 2011]. Foram também utilizados três itens da Escala de Motivação de Teste [Eklöf 2010] para se medir o Esforço. Após as medições, foram propostos alguns modelos para inferir o Esforço e Desempenho com base na estimação por Máxima Verossimilhança. Com as medidas de Importância, Interesse e Ansiedade, conseguiu-se explicar cerca de $60 \%$ da variância das medida de Esforço de realização do teste. Adicionando-se a medida de Probabilidade de Sucesso (expectativa), a melhora foi pouco significativa (2\% a mais). O fator mais relevante para o Esforço foi a importância dada ao teste. O modelo mais interessante para predição de desempenho utilizou todos os componentes (Importância, Interesse, Ansiedade, Probabilidade de Sucesso e Esforço) e conseguiu explicar aproximadamente 18\% da variância com os dados pré-teste e $28 \%$ com os dados pós-teste. Os fatores mais relevantes foram Esforço e Probabilidade de Sucesso, sendo que a primeira medida se manteve estável e a segunda se aprimorou no pós-teste.

Quando foi proposto o TAI, acreditava-se que o mesmo evitaria o tédio de alunos de alta habilidade e, de igual forma, frustração em pessoas com baixa habilidade [Weiss and Betz 1973]. Posteriormente, em 1976, os mesmos autores realizaram um estudo comparando um teste tradicional a um estratificado, que se tratava de uma implementação precursora do TAI atual, em que as questões eram separadas por estratos, ou categorias de dificuldade aferidas por um especialista. Eles descobriram uma melhora significativa da motivação, mas somente para estudantes de baixa habilidade. Já em outro trabalho [Eggen and Verschoor 2006], foi relatado que o TAI pode ser percebido como mais difícil, visto que os estudantes estão acostumados aos testes tradicionais com alta probabilidade de acerto. Em uma publicação mais recente [Ortner et al. 2013], foi observado um fato inesperado: em um TAI, os avaliados se sentiram mais satisfeitos após realizarem testes de menor dificuldade, apesar de terem obtido resultados piores do que aqueles de testes mais difíceis. 
V Congresso Brasileiro de Informática na Educação (CBIE 2016)

Anais do XXVII Simpósio Brasileiro de Informática na Educação (SBIE 2016)

Com base nisso, foi realizado um experimento com o objetivo de investigar o efeito motivacional do TAI [Ortner et al. 2014]. Em um grupo de 174 alunos de ensino médio, divididos em dois grupos, foi aplicado um teste de matriz [Hornke et al. 2000] nos modos adaptativo e tradicional. Após cinco minutos de prova, esta era interrompida e era medido o Fluxo [Csikszentmihalyi 2000], o Medo de Falhar e a Probabilidade de Sucesso, por meio dos questionários de [Vollmeyer and Rheinberg 2006], dentre estes, uma versão do QMC. Esperava-se encontrar maior Fluxo em um teste tradicional do que em um adaptativo (TAI). Os resultados mostraram que houve um maior Medo de Falhar e menor Probabilidade de Sucesso no TAI. Entretanto, a respeito do Fluxo, não houve diferenças significativas quando comparados os dois ambientes. Observou-se também que as maiores medidas de Fluxo corresponderam aos estudantes com melhores desempenhos.

Entretanto, esses trabalhos investigam contextos em larga escala. Este cenário diverge do habitual em sala de aula, em que se há poucas dezenas de alunos, pequena amostragem estatística e escassez de equipes especializadas em estatística e informática. No sentido prático, requisitos como facilidade de implementação ou calibração dinâmica são características relevantes a serem consideradas neste contexto. Neste intuito, a proposta de Teste Adaptativo Estratificado, apresentado a seguir, contribui para melhorar as avaliações sem despender grande esforço de implementação.

\subsection{O Teste Adaptativo Estratificado}

González-Sacristán e outros propuseram um método computacionalmente assistido para melhorar a qualidade das avaliações, aumentando a robustez, objetividade, segurança e relevância do conteúdo [González-Sacristán et al. 2013]. A abordagem, inspirada em TAI, divide as questões em categorias ordenadas pelo mais básico e fundamental até o mais específico e elaborado, que só é relevante após o domínio do conhecimento das categorias anteriores. Pretende-se que com este método, estudantes que investem em uma base fundamental sólida devam ser melhor recompensados do que aqueles que preferem partir para o avançado sem ter o domínio robusto dos fundamentos.

Foram apresentados dois modelos. No Modelo 1, cada estudante responde a um mesmo número de questões. Respostas corretas conduzem para o próximo nível; resposta incorretas, mantêm o aluno no mesmo nível. Esse caminhamento continua até que se responda um número determinado de questões. O nível da última questão e sua resposta indicam o resultado obtido pelo estudante. Já no Modelo 2, a quantidade de questões respondidas varia. De forma análoga, respostas corretas conduzem para níveis mais complexos e respostas incorretas, mantêm no mesmo nível. Entretanto, uma sequência de respostas erradas pode conduzir a um encerramento antecipado do teste. Assim, o mesmo pode ser encerrado por um número excessivo de respostas incorretas ou por atingir o limite máximo de questões a serem respondidas.

Para avaliar se o modelo é adequado na avaliação de habilidades de programação básica, foi realizado um estudo com aproximadamente 100 estudantes de graduação, em 2012 e 2013 [González-Sacristán et al. 2013]. Aplicaram dois testes: de completar lacunas e aberto, utilizando a metodologia proposta. Após removerem do estudo os estudantes que não executaram ambos os testes, restaram 30. Foi encontrado um coeficiente de Pearson de 0,66 e Spearman de 0,62 entre os resultados de ambas as provas, indicando que, dentro desta metodologia, as questões de completar, que são possíveis de serem computacionalmente assistidas, são um bom substitutivo das questões abertas. 
V Congresso Brasileiro de Informática na Educação (CBIE 2016)

Anais do XXVII Simpósio Brasileiro de Informática na Educação (SBIE 2016)

\section{Metodologia}

As atividades realizadas foram:

1. Seleção do grupo de teste

Como grupo de teste foram utilizadas duas turmas de disciplina de Redes de Computadores. Uma era do curso de Sistemas de Informação e a outra, de Engenharia da Computação, com 22 e 10 alunos, respectivamente.

2. Desenvolvimento da ferramenta

Foi desenvolvido um sistema próprio, com disponibilização web, escrito em Groovy/ Grails, que permitia aos próprios alunos se cadastrarem com acesso único (com login e senha). A ferramenta foi utilizada pelos alunos como preparação para as avaliações formativas convencionais, em complemento à lista de exercícios, em dois módulos. Para o primeiro módulo, o período disponível foi de uma semana antes da primeira prova. Para o segundo módulo, o período disponível foi de quatro dias antes da segunda prova. Nestes períodos, os alunos eram livres para se cadastrarem e acessarem o sistema quando lhes fosse conveniente, não sendo obrigatória sua participação.

3. Preparação do banco de questões

Considerando-se os assuntos do respectivo módulo, o professor da disciplina selecionou as questões aplicadas coletando-as de concursos públicos, do Exame Nacional de Desempenho dos Estudantes (ENADE), do Exame Nacional para Ingresso na Pós-Graduação em Computação (POSCOMP) e questões elaboradas por ele próprio. Estas questões tinham o formato de múltipla escolha e somente uma opção correta. Elas foram ordenadas nos graus de dificuldade: muito fácil, fácil, regular, difícil, muito difícil; tal que, a dificuldade muito fácil corresponde às questões com conceitos mais fundamentais e elementares da disciplina. Para o primeiro módulo, o banco de questões continha um único assunto com cinco, quatro, três, duas e uma questões, respectivamente, para cada nível de dificuldade. O segundo módulo também continha somente um assunto, mas com cinco, quatro, quatro, duas e uma questões, respectivamente, para os níveis de dificuldade.

4. Método de execução das questões

Para cada assunto, a execução das questões seguiu um método adaptativo estratificado [González-Sacristán et al. 2013], de forma que:

1. O estudante inicia recebendo aleatoriamente uma questão do primeiro nível: muito fácil.

2. O estudante responde a questão. Caso seja a quinta questão, o teste se encerra.

3. Caso acerte, recebe aleatoriamente uma questão do próximo nível, senão continua no mesmo nível. Repete-se o passo 2.

O Modelo 1 foi escolhido em detrimento do 2, pois a ferramenta tem por objetivo ser utilizada para estudo e preparação e não somente para avaliação. Deste modo, o modelo 2 apresentava um número menor de questões a serem executadas quando o estudante possuía baixa habilidade, retirando deste a oportunidade de se exercitar e alimentar sua curiosidade sobre a matéria.

5. Coleta de dados de motivação

Após a execução das questões do modelo [González-Sacristán et al. 2013], foi solicitado aos estudantes responderem o Questionário de Motivação Corrente 
V Congresso Brasileiro de Informática na Educação (CBIE 2016)

Anais do XXVII Simpósio Brasileiro de Informática na Educação (SBIE 2016)

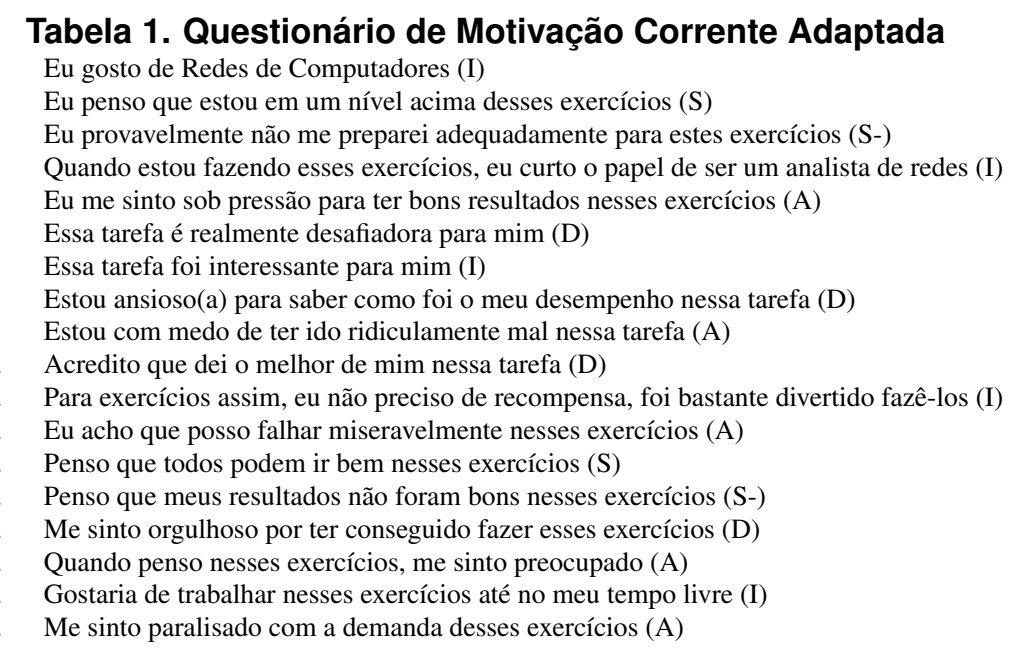

(QMC) [Vollmeyer and Rheinberg 2006] adaptado para a disciplina de Redes, a fim de se medir a Ansiedade (A), o Interesse (I), a Probabilidade de Sucesso Esperado (S) e o Desafio (D). As opções disponíveis seguiam a escala Likert de cinco opções, tal que 0 significa "discordo totalmente" e 4, "concordo totalmente". As perguntas do questionário estão listadas na Tabela 1.

6. Feedback para os alunos

Após responderem ao QMC, os estudantes tiveram acesso aos seus resultados, ao gabarito das questões realizadas e puderam responder as demais questões do banco que eram apresentadas para eles em ordem crescente de dificuldade. Este formato foi escolhido com base nos trabalhos da literatura [Yeh and Park 2015, Finley et al. 2011] que evidenciam um favorecimento da aprendizagem quando o feedback não é imediato e também quando a dificuldade desejada é ajustada, iniciando-se da atividade mais fácil para a mais difícil. Os resultados são sumarizados por assunto exibindo o número de questões corretas.

7. Análise dos dados

Cada fator foi representado pela média aritmética simples das respostas às questões do QCM correspondentes. Os dados foram analisados de duas formas. $\mathrm{Na}$ primeira, separou-se as amostras em três grupos distintos de acordo com o desempenho nas questões realizadas conforme o modelo adotado [González-Sacristán et al. 2013]. Foram verificados e comparados os resultados apresentados por cada grupo. Na segunda, foi aplicada a Correlação de Pearson entre as motivações medidas, o número de questões corretamente respondidas e o tempo total utilizado para a realização destas questões.

\section{Resultados}

Para o primeiro módulo, 17 estudantes se cadastraram no sistema (dois da turma de engenharia de computação, 15 de sistemas de informação). 15 responderam ao QMC, entretanto, três responderam todo o questionário com a opção neutro, sendo descartados da análise de motivação por não fornecerem nenhuma informação. Desses estudantes, 12 responderam todas as 15 questões disponibilizadas. Já no segundo módulo, 9 estudantes responderam ao QMC, sendo que três responderam todas as questões cadastradas, totalizando 18 estudantes distintos cadastrados e 16 que interagiram efetivamente. 
V Congresso Brasileiro de Informática na Educação (CBIE 2016)

Anais do XXVII Simpósio Brasileiro de Informática na Educação (SBIE 2016)

Na Tabela 2 são apresentados os valores médios dos fatores de motivação para três grupos de desempenho distintos: de zero a dois acertos, com três acertos e de quatro a cinco acertos. A Probabilidade de Sucesso, que teria um comportamento crescente, caso fosse um teste tradicional, apresentou um comportamento "ogival", em que o grupo 2 apresentou maiores valores, demonstrando que a metodologia estratificada teve efeito, porém não foi suficiente para demonstrar o esperado para um TAI (em que a percepção de sucesso seria bem próxima uma da outra). No segundo módulo (Tabela 3), os grupos apresentaram um comportamento crescente de Probabilidade de Sucesso. Entretanto, não é possível afirmar que esse comportamento é bem retratado, devido a pouca representatividade do grupo 3, com somente um estudante. Em comparação ao primeiro módulo, nota-se que os valores de Interesse aumentaram, apesar da participação ter sido mais baixa. Isso pode ter relação com o tempo em que o sistema ficou disponível (1 semana no $1^{\circ}$ módulo e 4 dias no $2^{\circ}$ módulo), ou que, dada a primeira experiência dos usuários, os menos interessados não se dispuseram a participar.

Tabela 2. Valores médios por grupo no $1^{\circ}$ módulo

\begin{tabular}{ccccccc}
\hline Grupo & $\#$ & Resultado & Ansiedade & P. de Sucesso & Interesse & Desafio \\
\hline 1 & 4 & $0-2$ & 1,45 & 1,37 & 1,6 & 1,625 \\
2 & 5 & 3 & 1,72 & 2,45 & 3 & 2,9 \\
3 & 3 & $4-5$ & 0,87 & 1,83 & 2,4 & 2,58 \\
\hline Total & 12 & $0-5$ & 1,4166 & 1,9375 & 2,3833 & 2,3958 \\
\hline
\end{tabular}

Tabela 3. Valores médios por grupo no $2^{\circ}$ módulo

\begin{tabular}{ccccccc}
\hline Grupo & $\#$ & Resultado & Ansiedade & P. de Sucesso & Interesse & Desafio \\
\hline 1 & 2 & $0-2$ & 2,1 & 1,75 & 2,2 & 2,25 \\
2 & 6 & 3 & 1,4 & 2,08 & 2,7 & 2,75 \\
3 & 1 & $4-5$ & 2,2 & 2,75 & 2,8 & 3 \\
\hline Total & 9 & $0-5$ & 1,6444 & 2,0833 & 2,6 & 2,6666 \\
\hline
\end{tabular}

Existem significativas causalidades entre Interesse e Resultado e maior ainda a participação da Probabilidade de Sucesso ao estimar o Resultado em testes convencionais [Penk and Schipolowski 2015]. Comparado a este trabalho, os resultados obtidos indicam a presença destas correlações, conforme a Tabela 4, mas se apresentam de forma fraca no segundo módulo, conforme a Tabela 5. No que se refere a Probabilidade de Sucesso, é um indício de que o método de seleção das questões, adaptativa, baseado no conhecimento tácito do professor, enfraqueça a relação com o Resultado, quando comparada aos testes convencionais. Visto que na metodologia adotada há um mecanismo de ajuste de dificuldade em que os mais hábeis tendem a ter uma prova mais difícil, a sensação de Sucesso do estudante não segue de maneira proporcional conforme o desempenho é melhor. Já a correlação Interesse e Resultado se apresenta de forma mais fraca pois os testes foram aplicados em um contexto em que o estudante está se esforçando para dominar a disciplina, enquanto que no estudo comparado se tratavam de avaliação após o ciclo de aprendizagem. Desta forma, foi menor o tempo em que o Interesse do 
V Congresso Brasileiro de Informática na Educação (CBIE 2016)

Anais do XXVII Simpósio Brasileiro de Informática na Educação (SBIE 2016)

estudante motiva o desenvolvimento de seu conhecimento, habilidades e competências. Ainda prosseguindo a comparação, um resultado equivalente em ambos os trabalhos foi que não houve significativa correlação entre a Ansiedade e o Resultado.

Fora da comparação, nota-se que as correlações mais presentes foram: Interesse e Probabilidade de Sucesso; Interesse e Desafio; Interesse e Tempo. Entende-se que o Interesse se relaciona com o otimismo do estudante frente ao teste, no desafio sentido por ele, e na quantidade de tempo que ele se dedica ao teste. Pelas medidas de Correlação de Pearson, não se pode afirmar a causalidade dos atributos, assim pode-se concluir que existem as seguintes possibilidades:

1. O Interesse influenciou o otimismo dos estudantes frente ao teste e/ou no desafio sentido por ele e/ou na quantidade de tempo que ele se dedicou ao teste.

2. O otimismo do estudante frente ao testes e/ou o desafio sentido por ele e/ou o tempo dedicado ao teste influenciou(aram) os estudantes a terem mais Interesse na disciplina.

3. Uma outra variável desconhecida influenciou o Interesse, a Probabilidade de Sucesso, o Desafio e o Tempo.

Por fim, foi observada uma correlação significativa entre Probabilidade de $\mathrm{Su}$ cesso e Tempo dedicado no primeiro módulo (Tabela 4), entretanto, o mesmo não se observou no segundo módulo (Tabela 5), indicando que este comportamento não pode ser tomado como padrão, mesmo para este desenho de experimento.

Tabela 4. Correlação de Pearson no $1^{\circ}$ módulo

\begin{tabular}{|c|c|c|c|c|c|}
\hline & Ansiedade & P. de Sucesso & Interesse & Desafio & Resultado \\
\hline Ansiedade & 1 & & & & \\
\hline P. de Sucesso & $-0,2872$ & 1 & & & \\
\hline Interesse & 0,0471 & $\mathbf{0 , 6 2 4 4}$ & 1 & & \\
\hline Desafio & 0,1831 & 0,0873 & $\mathbf{0 , 6 4 7 0}$ & 1 & \\
\hline Resultado & $-0,3970$ & $\mathbf{0 , 5 2 3 8}$ & $\mathbf{0 , 5 4 6 5}$ & 0,2720 & 1 \\
\hline Tempo & $-0,3380$ & $\mathbf{0 , 6 5 9 0}$ & 0,4147 & 0,0354 & 0,2706 \\
\hline
\end{tabular}

Tabela 5. Correlação de Pearson no $2^{\circ}$ módulo

\begin{tabular}{|c|c|c|c|c|c|}
\hline & Ansiedade & P. de Sucesso & Interesse & Desafio & Resultado \\
\hline Ansiedade & 1 & & & & \\
\hline P. de Sucesso & 0,1916 & 1 & & & \\
\hline Interesse & 0,4147 & $\mathbf{0 , 5 0 8 3}$ & 1 & & \\
\hline Desafio & 0,2801 & 0,3346 & $\mathbf{0 , 6 0 8 6}$ & 1 & \\
\hline Resultado & $-0,0763$ & 0,3985 & 0,3797 & 0,3807 & 1 \\
\hline Tempo & $-0,2058$ & 0,1416 & $\mathbf{0 , 5 0 2 9}$ & 0,1779 & $-0,4254$ \\
\hline
\end{tabular}

$16(50 \%)$ dos alunos interagiram com o sistema efetivamente, em algum momento. Este número é significativo, considerando o curto prazo de tempo concedido aos alunos e o fato de que a atividade não era pontuada. Após a realização dos dois módulos, em conversa com o professor em sala de aula, 11 alunos se manifestaram. Dentre esses, três manifestaram não ter usado o sistema, em nenhum momento, por falta de tempo e dois que, por falta de atenção, não viram o $e$-mail com as instruções de acesso ao sistema. Três 
V Congresso Brasileiro de Informática na Educação (CBIE 2016)

Anais do XXVII Simpósio Brasileiro de Informática na Educação (SBIE 2016)

deles manifestaram não terem usado o sistema no segundo módulo, por falta de tempo, que de fato foi curto. Dentre os seis que utilizaram o sistema, todos gostaram de utilizá-lo, o consideraram útil para estudar para a prova e fácil de usar. Esses resultados indicam a viabilidade de se utilizar o sistema como apoio à aprendizagem do aluno.

\section{Conclusões e Trabalhos Futuros}

Este artigo apresentou um estudo de caso da metodologia adaptativa estratificada de seleção de questões [González-Sacristán et al. 2013] em duas turmas da disciplina de Redes de Computadores. Os resultados apresentados indicaram que neste contexto nota-se uma correlação entre Probabilidade de Sucesso e Resultado, e entre Interesse e Resultado. Este resultado segue a mesma direção do documentado na literatura para avaliações tradicionais [Penk and Schipolowski 2015], entretanto se apresentam de maneira mais sutil, por influência do desenho deste experimento. Também de maneira equivalente, os resultados ratificam a ausência de relação entre a Ansiedade e o Resultado. Além da comparação, foi possível notar uma relação significativa e recorrente entre Interesse e Probabilidade de Sucesso, Interesse e Desafio, Interesse e Tempo de dedicação.

Além disso, notou-se que a metodologia estratificada apresentou efeito, porém não foi suficiente para demonstrar o esperado para um TAI. Estes resultados apoiam a recomendação da utilização desta metodologia em sala de aula como preparação dos estudantes para as avaliações formativas convencionais, fornecendo a eles feedback e consequentemente a oportunidade para se avaliar e reforçar os estudos em seus pontos fracos, visto que esta abordagem possui maior simplicidade de implementação do que o TAI baseado em TRI.

Os próximos passos do projeto são a utilização de um TAI baseado em TRI nas turmas de Redes do próximo semestre, utilizando a mesma metodologia de coleta de dados de motivação. O banco de questões será calibrado conforme a resposta dos estudantes deste semestre. A comparação entre estas metodologias servirá de base para a recomendação de uma metodologia a ser utilizada no desenvolvimento de testes adaptativos em sala de aula. Nota-se que no contexto geral de sala de aula, tem-se turmas com poucas dezenas de alunos e esta baixa amostragem dificulta a calibragem de parâmetros da TRI, possivelmente impactando na seleção adequada de questões. Nesse sentido, um método adaptativo estratificado, baseado somente na aferição do professor da disciplina, teria vantagens sobre um TAI baseado em TRI, além da facilidade de implementação? Se não, qual o tamanho da amostra necessária para que se tenha uma seleção de questões mais interessante que o estratificado? Medir, avaliar e comparar os fatores de motivação entre as metodologias adaptativas pode oferecer um caminho para responder a essas questões práticas.

\section{Referências}

Csikszentmihalyi, M. (2000). Beyond Boredom and Anxiety: Experiencing Flow in Work and Play. Jossey-Bass, 25th anniversary edition.

Eggen, T. J. M. and Verschoor, A. J. (2006). Optimal testing with easy or difficult items in computerized adaptive testing. applied psychological measurement. volume 30, pages 379-393. 
V Congresso Brasileiro de Informática na Educação (CBIE 2016)

Anais do XXVII Simpósio Brasileiro de Informática na Educação (SBIE 2016)

Eklöf, H. (2010). Student motivation and effort in the swedish timss advanced field study. In 4th IEA International Research Conference. Gothenburg.

Finley, J. R., Benjamin, A. S., Hays, M. J., Bjork, R. A., and Kornell, N. (2011). Benefits of accumulating versus diminishing cues in recall. Journal of Memory and Language, 64(4):289 - 298.

Freund, P. A. and Holling, H. (2011). Who wants to take an intelligence test? personality and achievement motivation in the context of ability testing. Personality and Individual Differences, 50(5):723 - 728 .

González-Sacristán, C., Molins-Ruano, P., Díez, F., Rodríguez, P., and Sacha, G. M. (2013). Computer-assisted assessment with item classification for programming skills. In Proceedings of the First International Conference on Technological Ecosystem for Enhancing Multiculturality, TEEM '13, pages 111-117, New York, NY, USA. ACM.

Hornke, L. F., Küppers, A., and Etzel, S. (2000). Design and evaluation of an adaptive matrices test. Diagnostica, 46:182-188.

Huang, S. X. (1996). A content-balanced adaptive testing. in computer aided learning and instruction in science and engineering. CALISCE-Computer Aided Learning and Instruction in Science and Engineering, pages 29-31.

Meijer, R. R. and Nering, M. L. (1999). Computerized adaptive testing: Overview and introduction. Applied Psychological Measurement, 23(3):187-194.

Ortner, T., Weißkopf, E., and Gerstenberg, F. (2013). Skilled but unaware of it: Cat undermines a test taker's metacognitive competence. European Journal of Psychology of Education, 28(1):37-51.

Ortner, T. M., Weißkopf, E., and Koch, T. (2014). I will probably fail: Higher ability students motivational experiences during adaptive achievement testing. European Journal of Psychological Assessment, 30:48-56.

Penk, C. and Schipolowski, S. (2015). Is it all about value? bringing back the expectancy component to the assessment of test-taking motivation. Learning and Individual Differences, 42:27 - 35 .

Piton-Gonçalves, J., Monzón, A. J. B., and Aluísio, S. M. (2009). Métodos de avaliação informatizada que tratam o conhecimento parcial do aluno e geram provas individualizadas. In Proceedings of the SBIE Conference on Simpósio Brasileiro de Informática na Educação, Florianópolis, SC, Brazil. Sociedade Brasileira de Computação.

Vollmeyer, R. and Rheinberg, F. (2006). Motivational effects on self-regulated learning with different tasks. Educational Psychology Review, 18(3):239-253.

Weiss, D. J. and Betz, N. E. (1973). Ability measurement: conventional or adaptive? Minneapolis, MN:University of Minnesota, Department of Psychology.

Yeh, D. D. and Park, Y. S. (2015). Improving learning efficiency of factual knowledge in medical education. Journal of Surgical Education, 72:882-889. 